\title{
The Kumaraswamy Gompertz distribution
}

\author{
Raquel C. da Silva ${ }^{\mathrm{a}}$, Jeniffer J. D. Sanchez ${ }^{\mathrm{b}}, \mathrm{F}^{\prime}$ abio P. Lima ${ }^{\mathrm{c}}$, Gauss M. Cordeiro ${ }^{\mathrm{d}}$ \\ Departamento de Estat'istica, \\ Universidade Federal de Pernambuco, \\ 50740-540, Recife, PE, \\ Brazil \\ ${ }^{a}$ e-mail:raquelmendiola@yahoo.com.br \\ ${ }^{b}$ e-mail:jjduartes@unal.edu.co \\ ${ }^{c}$ e-mail:fabio.p.l@hotmail.com \\ de-mail:gausscordeiro@uol.com.br \\ October 9, 2014
}

Abstract: We introduce the four-parameter Kumaraswamy Gompertz distribution. We obtain the moments, generating and quantilefunctions, Shannon and R'enyi entropies, mean deviations and Bonferroni and Lorenz curves. We provide a mixture representation for the density function of the order statistics. We discuss the estimation of the model parameters by maximum likelihood. We provide an application a real data set that illustrates the usefulness of the new model.

Key words: Maximum likelihood, Mean deviation, Moment, Survival data, Quantile function.

\section{Introduction}

The Gompertz model is a generalization of the exponential distribution and it is commonly used in many applied problems, particularly in lifetime data analysis. This model is considered for the analysis of survival data in some fields such as biology, computer and marketing science. If $Z$ has the Gompertz distribution with parameters $\theta>0$ and $\gamma>0$, denoted by $Z \sim G o(\theta, \gamma), Z$ has the cumulative distribution function (cdf) given by

$$
G_{\theta, \gamma}(z)=1-\exp \left\{-\frac{\theta}{\gamma}\left(e^{\gamma z}-1\right)\right\}, z>0
$$

and probability density function (pdf)

$$
g_{\theta, \gamma}(z)=\theta \exp \left\{\gamma z-\frac{\theta}{\gamma}\left(e^{\gamma z}-1\right)\right\}
$$

Note that the Gompertz distribution is a generalization of the exponential distribution, this is, equation (2) reduces to $\theta \exp (-\theta z)$ when $\gamma \rightarrow 0$. The properties of the Gompertz distribution 
have been studied by many authors in recent years. Pollard and Valkowincs (1992) were the first to study this distribution thoroughly. However, their results are true only in the case when the initial level of mortality is very close to zero. Kunimura (1998) obtained similar conclusions and determined the moment generating function (mgf) of $\mathrm{Z}$ is terms of the incomplete and complete gamma functions. Willemse and Koppelaar (2000) reformulated the Gompertz model to reforce mortality and derived relationships for this formulation. Willekens (2002) provided connections among the Gompertz, Weibull and type I extreme value distributions. Later, Marshall and Olkin (2007) described the negative Gompertz distribution. El-Gohary et al. (2013) proposed an extension of this distribution.

In this paper, we study a new four-parameter model called the Kumaraswamy Gompertz ("KwGo" for short) distribution. The paper is organized as follows. In Section 2, we define the density and failure rate functions of the KwGo distribution. In Sections 3 to 8, a range of mathematical properties in terms of the proposed model is investigated. These include the density expansion, moments, mgf, Shannon and Rényi entropies, mean deviations, Bonferroni and Lorenz curves, quantile function and some properties of the order statistics. In Section 9, we present the estimation procedure using the method of maximum likelihood. An application of the new model to a real data set is illustrated in Section 10. Finally, some concluding remarks are given in Section 11.

\section{The KwGodistribution}

The Kumaraswamy (Kw) model introduced by Kumaraswamy (1980) is a two-parameter distribution on the interval $(0,1)$ whose cdf is given by

$$
\Pi(x ; a, b)=1-\left(1-x^{a}\right)^{b}, x \in(0,1),
$$

where $a>0$ and $b>0$ are shape parameters. The pdf corresponding to (3) is

$$
\pi(x ; a, b)=a b x^{a-1}\left(1-x^{a}\right)^{b-1}, x \in(0,1) .
$$

The reader is referred to Jones (2009) for further details on the Kw distribution.

For any baseline cumulative function $G(x)$ and density function $g(x)=d G(x) / d x$, Cordeiro and de Castro (2011) proposed the Kumaraswamy $\mathrm{G}$ (" $K w G$ " for short) distribution with pdf $f(x)$ and cdf $F(x)$ given by

$$
f(x)=a b g(x) G^{a-1}(x)\left\{1-G^{a}(x)\right\}^{b-1}
$$

and

$$
F(x)=1-\left\{1-G^{a}(x)\right\}^{b},
$$


respectively. The $K w G$ distribution has the same parameters of the $G$ distribution plus two ad- ditional shape parameters $a>0$ and $b>0$. For $a=b=1$, the $G$ distribution is a basic exemplar of the $K w G$ distribution with a continuous crossover towards cases with different shapes (e.g., a particular combination of skewness and kurtosis). The $K w G$ family of densities (4) allows for greater flexibility of its tails and can be widely applied in many areas of biology and engineering. For a detailed survey of this family, the reader is referred to Cordeiro and de Castro (2011) and Nadarajah et al. (2012).

The four-parameter $K w G o$ cdf is defined from (5) by taking $G(x)$ to be equal to the cdf (1). Then, the KwGo cdf becomes

$$
F(x)=1-\left[1-\left(1-\exp \left\{-\frac{\theta}{\gamma}\left(e^{\gamma x}-1\right)\right\}\right)^{a}\right]^{b}
$$

Here, we have three positive shape parameters $\theta, a$ and $b$ and a positive scale parameter $\gamma$. The pdf and the hazard rate function (hrf) corresponding to (6) (for $x>0$ ) are given by

$$
\begin{gathered}
f(x)=a b \theta \\
\exp \left\{\gamma x-\frac{\theta}{\gamma}\left(e^{\gamma x}-1\right)\right\}\left[1-\exp \left\{-\frac{\theta}{\gamma}\left(e^{\gamma x}-1\right)\right\}\right]^{a-1} \\
\times\left[1-\left(1-\exp \left\{-\frac{\theta}{\gamma}\left(e^{\gamma x}-1\right)\right\}\right)^{a}\right]^{b-1}
\end{gathered}
$$

and

$$
h(x)=\frac{a b \theta \exp \left\{\gamma x-\frac{\theta}{\gamma}\left(e^{\gamma x}-1\right)\right\}\left[1-\exp \left\{-\frac{\theta}{\gamma}\left(e^{\gamma x}-1\right)\right\}\right]^{a-1}}{1-\left(1-\exp \left\{-\frac{\theta}{\gamma}\left(e^{\gamma x}-1\right)\right\}\right)^{a}}
$$

respectively. Figures 1 and 2 display some plots of the pdf and hrf of the proposed distribution for some parameter values. 
$\mathrm{KwGo}(\mathrm{a}, 0.5,0.5,0.1)$

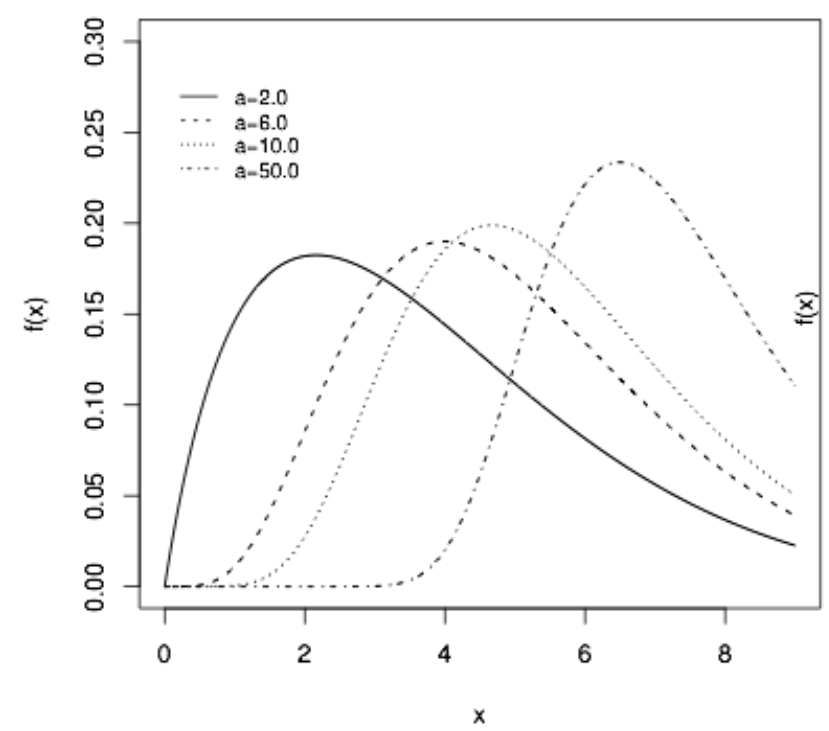

$\mathrm{KwGo}(2, \mathrm{~b}, 0.5,0.1)$

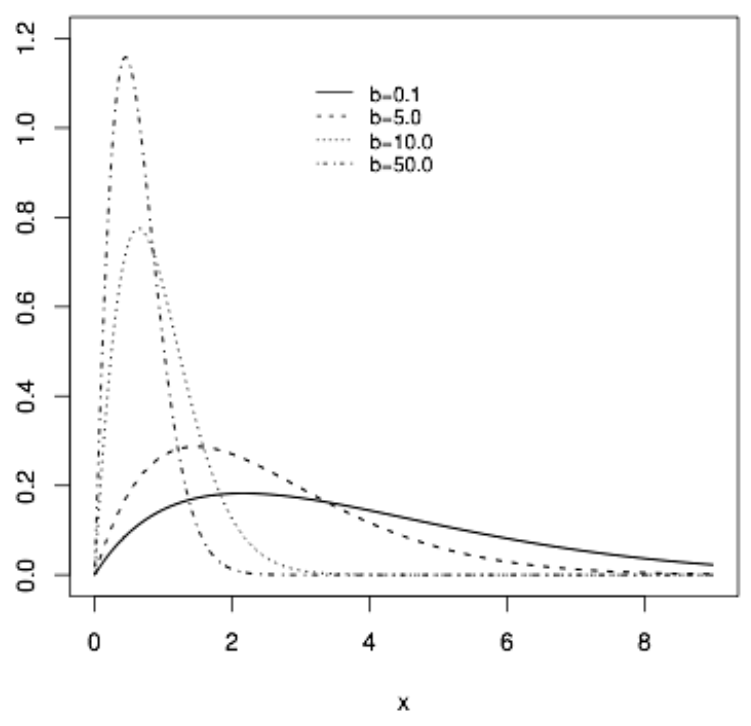

Figure 1: Plots of the pdf (7) for some parameter values. 
KwGo(a, 5, 0.5, 0.1)

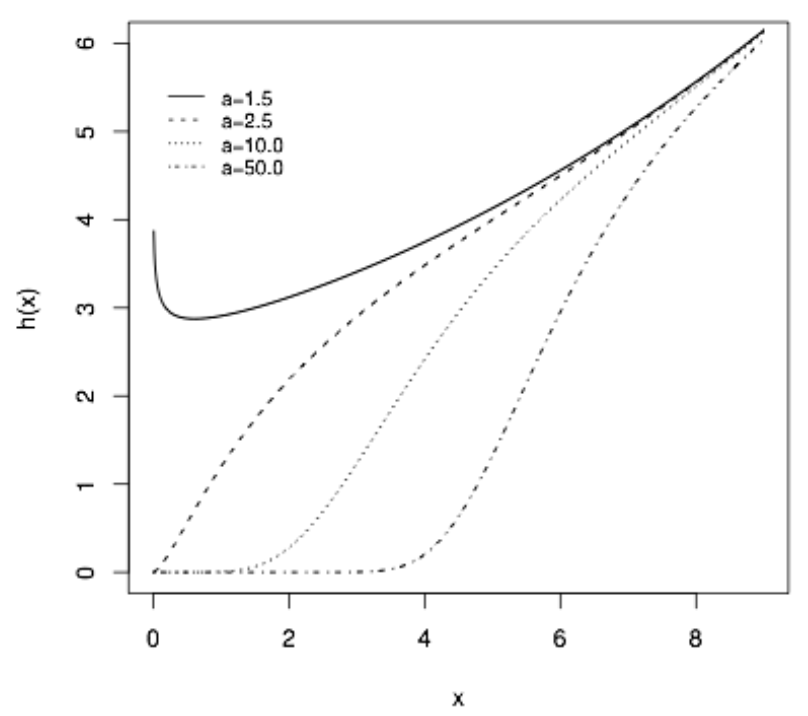

$\mathrm{KwGo}(2, \mathrm{~b}, 1,0.1)$

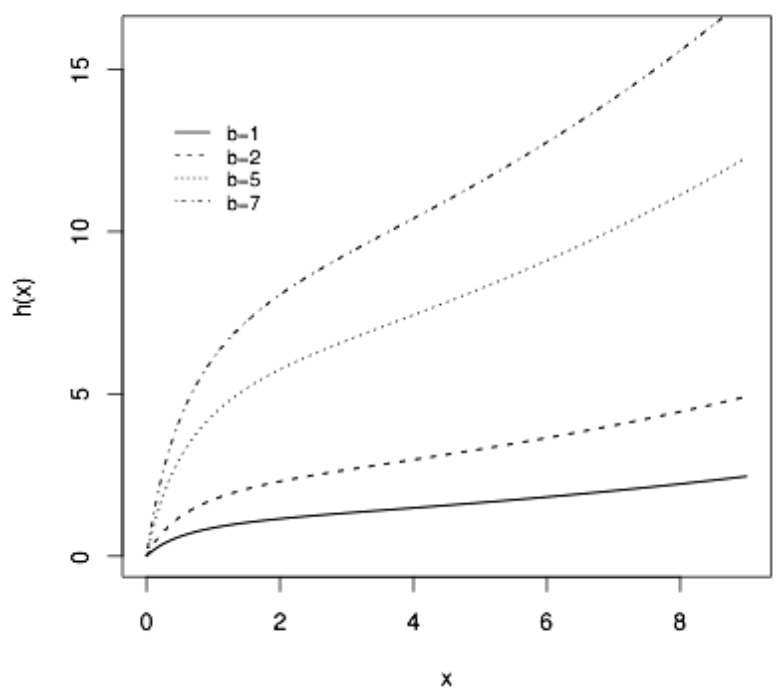

Figure 2: Plots of the hrf (8) for some parameter values.

Hanceforth, a random variable $X$ having density function (7) is denoted

$$
X \sim K w G o(a, b, \theta, \gamma)
$$

\section{Density expansion}

Equations (6) and (7) are straightforward to compute using modern computer resources with analytic and numerical capabilities. However, we can express $F(x)$ and $f(x)$ in terms of infinite weighted sums of cdf's and pdf's of the Go distributions. Using the power series for $|z|<1$ and $\alpha>0$

$$
(1-z)^{\alpha}=\sum_{j=0}^{\infty}(-1)^{j}\left(\begin{array}{l}
\alpha \\
j
\end{array}\right) z^{j}
$$

we can rewrite $F(x)$ as

$$
F(x)=1-\sum_{k=0}^{\infty}(-1)^{k}\left(\begin{array}{l}
b \\
k
\end{array}\right)\left[1-\exp \left\{-\frac{\theta}{\gamma}\left(e^{\gamma x}-1\right)\right\}\right]^{k a} .
$$

After some algebra, we obtain 


$$
F(x)=\sum_{j=0}^{\infty} t_{j} G_{(j+1) \theta, \gamma}(x)
$$

where (for $j \geq 0$ )

$$
t_{j}=t_{j}(a, b)=\sum_{k=0}^{\infty}(-1)^{k+j}\left(\begin{array}{c}
b \\
k+1
\end{array}\right)\left(\begin{array}{c}
(k+1) a \\
j+1
\end{array}\right)
$$

and $G_{(j+1) \theta, \gamma}(x)$ is the $G o$ cdf with parameters $(j+1) \theta$ and $\gamma$. By differentiating (9), the density function of $X$ can be expressed as

$$
f(x)=\sum_{j=0}^{\infty} t_{j} g_{(j+1) \theta, \gamma}(x)
$$

where $g_{(j+1) \theta, \gamma}(x)$ is the Go pdf with parameters $(j+1) \theta$ and $\gamma$.

Mathematical properties for the KwGo distribution can be obtained from equation (11) and those of the Go distribution.

\section{Moments and Generating function}

The $n$-th ordinary moment of $X$ is given by

$$
E\left(X^{n}\right)=\sum_{j=0}^{\infty} t_{j} E\left(Y_{j}^{n}\right)
$$

where $Y_{j} \sim \operatorname{Go}(\theta(j+1), \gamma)$. The $n$-th moment of $Y_{j}$ is given by

$$
E\left(Y_{j}^{n}\right)=\frac{n !}{\gamma^{n}} e^{(j+1)^{\theta} / \gamma} E_{1}^{n-1}\left(\frac{(j+1) \theta}{\gamma}\right)
$$

where

$$
E_{1}^{n-1}(z)=\sum_{k=1}^{\infty} \frac{1}{(-k)^{n}} \frac{(-z)^{k}}{k !}+\frac{(-1)^{n}}{n !} \sum_{k=0}^{\infty}\left(\begin{array}{l}
n \\
k
\end{array}\right) \log (z)^{n-1} \Psi_{k} .
$$

Here the first term is a power series of the generalized integral-exponential function (Milgram,1985) and

$$
\Psi_{n}=\lim _{t \rightarrow 0} \sum_{l=0}^{n-1}\left(\begin{array}{c}
n-1 \\
l
\end{array}\right) \Gamma(1-t)^{n-1-l} \psi^{n-1}(1-t),
$$


where $\psi^{n}(z)=\frac{d^{n}}{d z^{n}} \psi(z)$ denotes the polygamma function. So $\mathrm{E}\left(X^{n}\right)$ reduces to

$$
E\left(X^{n}\right)=\frac{n !}{\gamma^{n}} \sum_{j=0}^{\infty} t_{j} e^{(j+1)^{\theta} / \gamma} E_{1}^{n-1}\left(\frac{(j+1) \theta}{\gamma}\right) .
$$

The mgf of $X$ can be expressed from (11) as a linear combination of the mgf's of the Go distributions as follows

$$
M_{X}(t)=\sum_{j=0}^{\infty} t_{j} M_{(j+1) \theta, \gamma}(t),
$$

where $M_{(j+1) \theta, \gamma}(t)$ is the Go mgf with parameters $(j+1) \theta$ and $\gamma$ given by

$$
M_{(j+1) \theta, \gamma}(t)=\frac{(j+1) \theta}{\gamma} e^{(j+1)^{\theta} / \gamma} E_{t / \gamma}\left(\frac{(j+1) \theta}{\gamma}\right),
$$

where

$$
E_{t / \gamma}\left(\frac{(j+1) \theta}{\gamma}\right)=\left(\frac{(j+1) \theta}{\gamma}\right)^{\frac{t}{\gamma}-1} \Gamma\left(1-\frac{t}{\gamma}, \frac{(j+1) \theta}{\gamma}\right)
$$

and $\Gamma(c, x)=\int_{x}^{\infty} v^{c-1} e^{-v} d v$ is the complementary incomplete gamma function.

\section{Quantile function}

The $K w G o$ quantile function, say $Q(u)=F^{-1}(u)$, is given by

$$
x=Q(u)=\frac{1}{\gamma} \log \left[1-\frac{\gamma}{\theta} \log \left(1-\left[1-(1-u)^{\frac{1}{b}}\right]^{\frac{1}{a}}\right)\right],
$$

where $u \in(0,1)$.

The effect of the shape parameters $a$ and $b$ on the skewness and kurtosis of the new distribution can be considered based on quantile measures. The shortcomings of the classical skewness and kurtosis measures are well-known. One of the earliest skewness measures to be suggested is the Bowley skewness (Kenney and Keeping, 1962) given by

$$
B=\frac{Q(3 / 4)+Q(1 / 4)-Q(1 / 2)}{Q(3 / 4)-Q(1 / 4)} .
$$

Since only the middle two quartiles are considered and the outer two quartiles are ignored, this adds robustness to the measure. The Moors kurtosis is based on octiles 


$$
M=\frac{Q(7 / 8)-Q(5 / 8)+Q(3 / 8)-Q(1 / 8)}{Q(6 / 8)-Q(2 / 8)} .
$$

The measures $B$ and $M$ are less sensitive to outliers and they exist even for distributions without moments. In Figures 3 and 4, we plot the measures $B$ and $M$ for the KwGo distribution as functions of $a$ and $b$ for fixed values of the other parameters.

\section{Mean Deviations}

The mean deviations of $X$ about the mean $\delta_{1}$ and about the median $\delta_{2}$ are given by

$$
\delta_{1}=E(|X-\mu|)=2 \mu F(\mu)-2 T(\mu) \text { and } \delta_{2}=E(|X-M|)=\mu-2 T(M),
$$

respectively, where $\mu=\mathrm{E}(\mathrm{X})$ and $M=\operatorname{median}(\mathrm{X})$ is given by

$$
M=\gamma^{-1} \log \left[1-\log \left[1-\left(1-2^{-1 / b}\right)^{1 / a}\right] \theta^{-1} \gamma\right],
$$

KwGo(a,b,50,0.5)

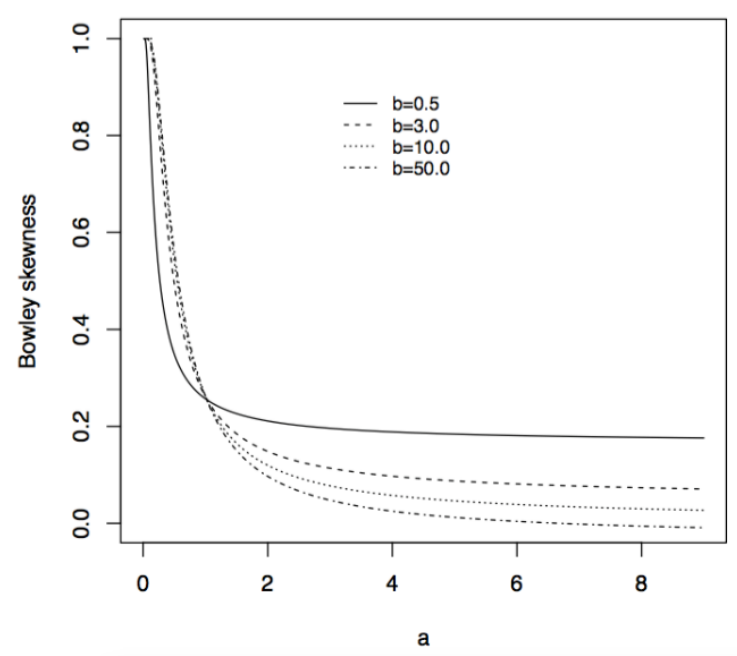

KwGo(a,b,1,10)

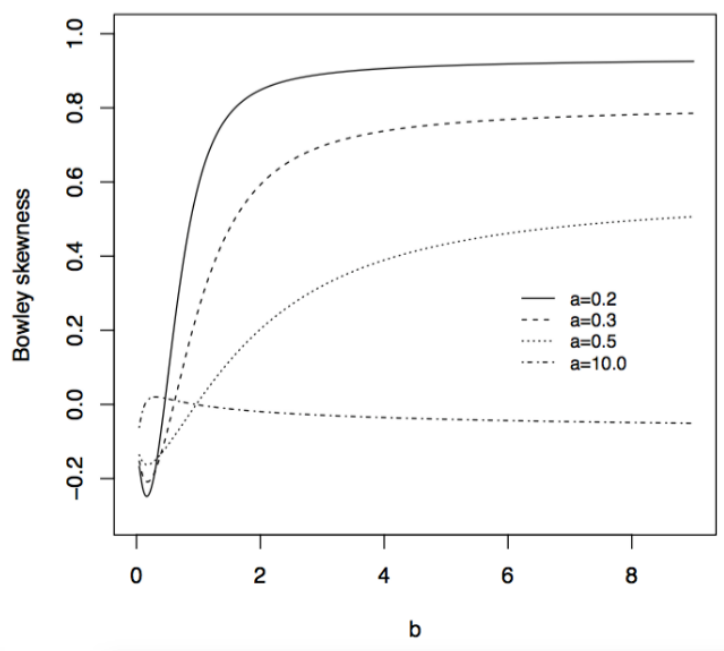

Figure 3: (a) Skewness of $X$ as function of $a$ for some values of $b$ and (b) skewness of $X$ as function of $b$ for some values of $a$. 

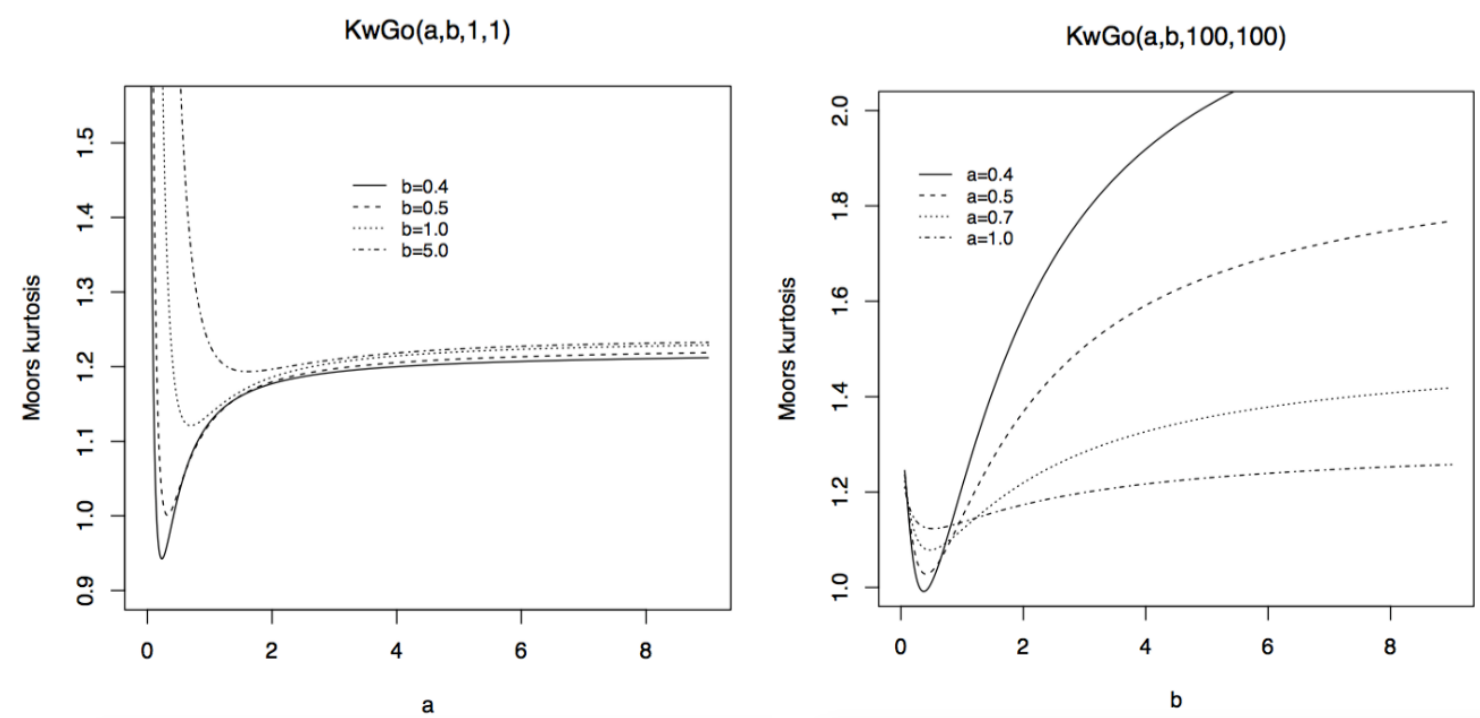

Figure 4: (a) Kurtosis of $X$ as function of $a$ for some values of $b$ and (b) kurtosis of $X$ as function of $b$ for some values of $a$.

$\mathrm{F}(\mu)$ comes from $(6)$ and $\mathrm{T}(\mathrm{z})$ is given by

$$
T(z)=\sum_{i=0}^{\infty} w_{i} J_{i}(z)
$$

where

$$
J_{i}(z)=(i+1) \sum_{j, k=0}^{\infty} \frac{-1^{k+j} a \theta^{k+1}\left[1+e^{(k+1) \gamma z}\{(k+1) \gamma z-1\}\right]}{(j+1)^{-k} \gamma^{k+2}(k+1)^{2} k !}\left(\begin{array}{c}
(i+1) a-1 \\
j
\end{array}\right) .
$$

Equation (13) can be used to determine Bonferroni and Lorenz curves. They are defined for a given probability $p$ by $B(p)=\mathrm{T}(\mathrm{q}) /(\mathrm{p} \mu)$ and $L(p)=\mathrm{T}(\mathrm{q}) / \mu$, respectively, where

$$
q=\gamma^{-1} \log \left[1-\log \left[1-\left(1-(1-p)^{-1 / b}\right)^{1 / a}\right] \theta^{-1} \gamma\right]
$$




\section{Order Statistics}

The order statistics and their moments are one of the most fundamental tools in nonparametric statistics and inference. The pdf and cdf of the $i$-th order statistic, say $X_{i: n}$, are given by

$$
f_{i: n}(x)=\frac{1}{B(i, n-i+1)} \sum_{s=0}^{n-i}(-1)^{s}\left(\begin{array}{c}
n-i \\
s
\end{array}\right) f(x) F(x)^{i+s-1}
$$

and

$$
F_{i: n}(x)=\frac{1}{B(i, n-i+1)} \sum_{s=0}^{n-i} \frac{(-1)^{s}}{i+s}\left(\begin{array}{c}
n-i \\
s
\end{array}\right) F(x)^{i+s}
$$

\subsection{Probability density and cumulative distribution functions}

Let $X_{1}, \ldots, X_{n}$ be a random sample of size $n$ from the $\operatorname{Kw} G o(a, b, \theta, \gamma)$ model. Then, the pdf and cdf of the $i$-th order statistic can be obtained from (14) and (15) by setting $F^{i+s}(x)=$ $\left[\sum_{k=0}^{\infty}(-1)^{k}\left(\begin{array}{c}b \\ k+1\end{array}\right) G^{(k+1) a}(\mathrm{x})\right]^{i+s}$. From now on, we use an equation by Gradshteyn and Ryzhik (2000, Section 3.14) for a power series raised to a positive integer $n$

$$
\left(\sum_{r=0}^{\infty} w_{r} u^{r}\right)^{n}=\sum_{r=0}^{\infty} c_{n, r} u^{r}
$$

where the coefficients $c_{n, r}$ (for $r=1,2, \ldots$ ) are determined from the recurrence equation

$$
c_{n, r}=\left(r w_{0}\right)^{-1} \sum_{j=1}^{r}[j(n+1)-r] w_{j} c_{r, r-j},
$$

and $c_{n, 0}=w_{0}^{n}$. So, equations (14) and (15) can be expressed as 


$$
F_{i: n}(x)=\frac{1}{B(i, n-i+1)} \sum_{s=0}^{n-i} \sum_{k, m=0}^{\infty} \frac{(-1)^{m+s}}{(i+s)}\left(\begin{array}{c}
n-i \\
s
\end{array}\right)\left(\begin{array}{c}
a(k+i+s) \\
m+1
\end{array}\right) c_{i+s, k} G_{(m+1) \theta, \gamma}(x)
$$

and

$$
f_{i: n}(x)=\frac{1}{B(i, n-i+1)} \sum_{s=0}^{n-i} \sum_{k, m=0}^{\infty} \frac{(-1)^{m+s}}{(i+s)}\left(\begin{array}{c}
n-i \\
s
\end{array}\right)\left(\begin{array}{c}
a(k+i+s) \\
m+1
\end{array}\right) c_{i+s, k} g_{(m+1) \theta, \gamma}(x)
$$

The last equation reveals that the pdf of $X_{i: n}$ can be given as a mixture of Go densities. The structural properties of $X_{i: n}$ are then easily obtained from those of the Go distribution.

\subsection{Moments}

The 1-th moment of $X_{i: n}$ follows as

$$
\begin{gathered}
E\left(X_{i: n}^{l}\right)=\frac{1}{B(i, n-i+1)} \sum_{s=0}^{n-i} \sum_{k, m=0}^{\infty} \frac{(-1)^{m+s} c_{i+s, k}}{(i+s)}\left(\begin{array}{c}
n-i \\
s
\end{array}\right)\left(\begin{array}{c}
a(k+i+s) \\
m+1
\end{array}\right) \int_{0}^{\infty} x^{l} g_{(m+1) \theta, \gamma}(x) d x \\
=\frac{1}{B(i, n-i+1)} \sum_{s=0}^{n-i} \sum_{k, m=0}^{\infty} \frac{(-1)^{m+s} c_{i+s, k}}{(i+s)}\left(\begin{array}{c}
n-i \\
s
\end{array}\right)\left(\begin{array}{c}
a(k+i+s) \\
m+1
\end{array}\right) \times \frac{l !}{\gamma^{l}} e^{(m+1) \theta / \gamma} \boldsymbol{E}_{1}^{l-1}\left(\frac{(m+1) \theta}{\gamma}\right) .
\end{gathered}
$$




\section{Shannon and Rényi Entropy}

The entropy of a random variable $X$ with density function $f(x)$ is a measure of variation of the uncertainty. The Shannon entropy is defined by Shannon (1948) as

$$
S[f(x)]=E(\log [f(x)])
$$

The Shannon entropy of $X$ is determined as

$$
S[f(x)]=-\log (a b \theta)-\gamma E(x)+\frac{\left[M_{x}(\gamma)-1\right] \theta}{\gamma}+\frac{(a-1)[C+\varphi(b+1)]}{a}-\frac{(b-1)}{b},
$$

where $C$ is the Euler's constant and $\varphi(\cdot)$ is the digamma function.

Another popular entropy measure is the Rényi entropy defined by Rényi (1961) given by

$$
R(c)=\frac{1}{1-c} \log \left(\int_{-\infty}^{\infty} f^{c}(x)\right), c>0, c \neq 1 .
$$

The Rényi entropy of $X$ is given by

$$
\begin{gathered}
R(c)=\frac{c}{1-c} \log \gamma+\frac{2-c}{1-c} \log \theta+ \\
\frac{1}{1-c} \log \left[\sum_{j, k=0}^{\infty}(-1)^{k+j+1}(c+k)^{-c} e^{(k+c) \theta / \gamma}\left(\begin{array}{c}
(b-1) c \\
j
\end{array}\right)\left(\begin{array}{c}
(c+j) a-c \\
k
\end{array}\right) \Gamma\left(c, \frac{(k+c) \theta}{\gamma}\right)\right]
\end{gathered}
$$




\section{Estimation}

We consider estimation of the parameters of the KwGo distribution by the method of maximum likelihood. Let $x=\left(x_{1}, \ldots, x_{n}\right)^{T}$ be a sample of size $n$ from the $K w G o$ distribution with unknown parameter vector $\Theta=(a, b, \theta, \gamma)^{T}$. The total log-likelihood function for $\Theta$ is

$$
\ell(\Theta)=\log (a b \theta)+\gamma x-\frac{\theta}{\gamma}\left(e^{\gamma x}-1\right) b+(a-1) \log \left[1-\exp \left\{-\frac{\theta}{\gamma}\left(e^{\gamma x}-1\right)\right\}\right]
$$

The log-likelihood can be maximized either directly or by solving the nonlinear likelihood equations obtained by differentiating (16). We obtain the maximum likelihood estimates (MLEs) using the components of the score vector $U(\Theta)$ given by

$U_{a}(\Theta)=\frac{\partial \ell(\Theta)}{\partial a}=\frac{1}{a}+\log \left[1-\exp \left\{-\frac{\theta}{\gamma}\left(e^{\gamma x}-1\right)\right\}\right]$

$U_{b}(\Theta)=\frac{\partial \ell(\Theta)}{\partial b}=\frac{1}{b}-\frac{\theta}{\gamma}\left(e^{\gamma x}-1\right)$

$U_{\theta}(\Theta)=\frac{\partial \ell(\Theta)}{\partial \theta}=\frac{1}{\theta}-\frac{\theta\left(e^{\gamma x}-1\right)}{\gamma}\left\{b+(a-1) \frac{\exp \left\{-\frac{\theta}{\gamma}\left(e^{\gamma x}-1\right)\right\}}{\left[1-\exp \left\{-\frac{\theta}{\gamma}\left(e^{\gamma x}-1\right)\right\}\right]}\right\}$,

$U_{\gamma}(\Theta)=\frac{\partial \ell(\Theta)}{\partial \gamma}=x+\left[\frac{\left(e^{\gamma x}-1\right)}{\gamma}-x e^{\gamma x}\right]\left\{\frac{b \theta}{\gamma}-\frac{(a-1) \theta}{\gamma} \frac{\exp \left\{-\frac{\theta}{\gamma}\left(e^{\gamma x}-1\right)\right\}}{\left[1-\exp \left\{-\frac{\theta}{\gamma}\left(e^{\gamma x}-1\right)\right\}\right]}\right\}$.

For interval estimation and hypothesis tests on the model parameters, we require the observed information matrix. The $4 \times 4$ unit observed information matrix $J=J_{n}(\Theta)$ is determined by

$$
J=-\left[\begin{array}{cccc}
J_{a a} & J_{a b} & J_{a \theta} & J_{a \gamma} \\
J_{b a} & J_{b b} & J_{b \theta} & J_{b \gamma} \\
J_{\theta a} & J_{\theta b} & J_{\theta \theta} & J_{\theta \gamma} \\
J_{\gamma a} & J_{\gamma b} & J_{\gamma \theta} & J_{\gamma \gamma}
\end{array}\right]
$$

whose elements are given in the Appendix. 


\section{Application}

We emphasize the flexibility of the new distribution by means of a real data set and fit the Go, exponentiated Gompertz (ExpGo), beta Gompertz (BGo) and KwGo distributions.

The cdf of the ExpGo distribution is given by

$$
H_{a}(x)=\left[1-\exp \left\{-\frac{\theta}{\gamma}\left(e^{\gamma x}-1\right)\right\}\right]^{a}
$$

and the pdf reduces to (for a positive power $a>0$ )

$$
h_{a}(x)=a \theta \exp \left\{\gamma x-\frac{\theta}{\gamma}\left(e^{\gamma x}-1\right)\right\}\left[1-\exp \left\{-\frac{\theta}{\gamma}\left(e^{\gamma x}-1\right)\right\}\right]^{a-1} .
$$

Eugene et al. (2002) defined the beta class of distributions. The BGo pdf can be expressed as

$$
f(x)=\frac{\theta \exp \left\{\gamma x-\frac{\beta \theta}{\gamma}\left(e^{\gamma x}-1\right)\right\}}{B(\alpha, \beta)}\left[1-\exp \left\{-\frac{\theta}{\gamma}\left(e^{\gamma x}-1\right)\right\}\right]^{\alpha-1}
$$

where $B(\alpha, \beta)=\Gamma(\alpha) \Gamma(\beta) / \Gamma(\alpha+\beta)$ is the beta function.

The data are the proportions of HIV-infected people in 137 countries (Rushton and Templer, 2009). The MLEs of the unknown parameters (standard errors in parentheses) of the fitted models are given in Table 1. Further, the values of the statistics AIC (Akaike Information Criterion), AICC (Akaike Information Criterion with Correction) and BIC (Bayesian Information Criterion) are calculated for the KwGo,BGo,ExpGo and Go distributions. The Cramér-von Mises and Anderson-Darling (W and A for short) statistics are calculated for the KwGo,ExpGo and Go models. The computations are performed using the AdequacyModel package in R. Based on the values of these statistics, we can conclude that the KwGo model is better than the other distributions to fit these data. 
Table 1: MLEs and goodness-of-fit statistics

\begin{tabular}{|c|c|c|c|c|c|c|c|c|c|}
\hline Models & $a$ & $\mathbf{b}$ & $\theta$ & $\gamma$ & AIC & AICC & BIC & $W$ & $\mathrm{~A}$ \\
\hline KwGo & $\begin{array}{c}0.477 \\
(0.050)\end{array}$ & $\begin{array}{c}7.535 \\
(3.057)\end{array}$ & $\begin{array}{c}0.010 \\
(0.008)\end{array}$ & $\begin{array}{c}0.000 \\
(0.024)\end{array}$ & 262.600 & 263.040 & 272.858 & 1.245 & 7.479 \\
\hline BGo & $\begin{array}{c}0.374 \\
(0.055)\end{array}$ & $\begin{array}{c}4.645 \\
(6.053) \\
\end{array}$ & $\begin{array}{c}0.033 \\
(0.047) \\
\end{array}$ & $\begin{array}{c}0.000 \\
(0.020)\end{array}$ & 284.434 & 284.873 & 294.691 & 1.503 & 8.810 \\
\hline ExpGo & $\begin{array}{c}0.363 \\
(0.054)\end{array}$ & $\begin{array}{c}1.000 \\
-\end{array}$ & $\begin{array}{c}0.173 \\
(0.061)\end{array}$ & $\begin{array}{c}0.000 \\
(0.020)\end{array}$ & 285.95 & 286.210 & 293.643 & 1.539 & 8.993 \\
\hline Go & $\begin{array}{c}1.000 \\
-\end{array}$ & $\begin{array}{c}1.000 \\
-\end{array}$ & $\begin{array}{c}0.057 \\
(0.057)\end{array}$ & $\begin{array}{c}0.010 \\
(0.010)\end{array}$ & 376.356 & 376.485 & 381.485 & 1.543 & 9.009 \\
\hline
\end{tabular}

Figure 5 displays the histogram of the data and the four fitted KwGo, BGo, ExpGo and Go densities. We can verify that the KwGo distribution provides an adequate fit to these data.

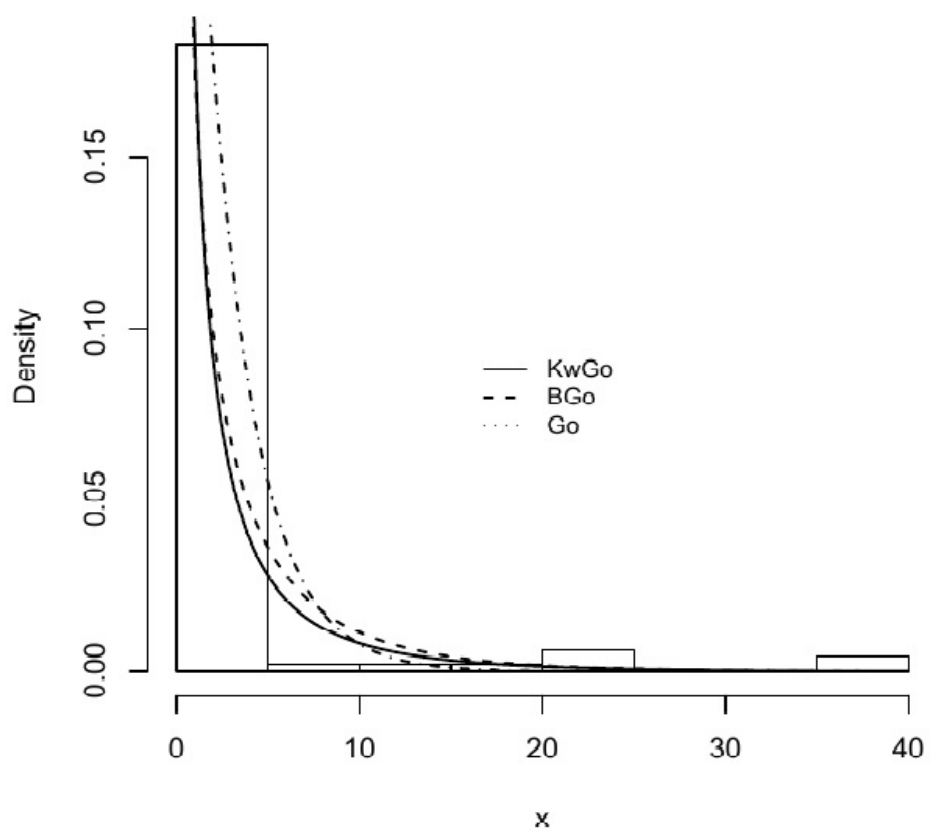

Figure 5: Plots of the fitted models to the current data. 


\section{Concluding remarks}

We study a new four-parameter model named the Kwmaraswamy Gompertz distribution. We provide the moments, generating function, Shannon and Rényi entropies, mean deviations, Bonferroni and Lorenz curves and the moments of the order statistics. We discuss the estimation of the parameters by maximum likelihood. One application of the new distribution is given to prove its flexibility to fit real lifetime data.

\section{Appendix}

The elements of the unit observed information matrix $J=J_{n}(\Theta)$ are

$$
\begin{array}{ll}
J_{a a}=\frac{\partial^{2} \log }{\partial a^{2}}=-\frac{1}{a^{2}}, & J_{b b}=\frac{\partial^{2} \log }{\partial b^{2}}=-\frac{1}{b^{2}}, \\
J_{a \theta}=\frac{\partial^{2} \log }{\partial a \partial \theta}=-\frac{\left(e^{\gamma x}-1\right)}{\gamma} \frac{\exp \left\{-\frac{\theta}{\gamma}\left(e^{\gamma x}-1\right)\right\}}{\left[1-\exp \left\{-\frac{\theta}{\gamma}\left(e^{\gamma x}-1\right)\right\}\right]}, \quad J_{b \theta}=\frac{\partial^{2} \log }{\partial b \partial \theta}=-\frac{\left(e^{\gamma x}-1\right)}{\gamma}, J_{a \gamma}= \\
\frac{\partial^{2} \log }{\partial a \partial \gamma}=\left(\frac{e^{\gamma x}(x \gamma-1)+1}{\gamma^{2}}\right) \frac{\exp \left\{-\frac{\theta}{\gamma}\left(e^{\gamma x}-1\right)\right\} \theta}{\left[1-\exp \left\{-\frac{\theta}{\gamma}\left(e^{\gamma x}-1\right)\right\}\right]}, \quad J_{b \gamma}=\frac{\partial^{2} \log }{\partial b \partial \gamma}=-\frac{e^{\gamma x}(x \gamma-1)+1}{\gamma^{2} \theta^{-1}}, \\
J_{\theta a}=\frac{\partial^{2} \log }{\partial \theta \partial a}=\frac{\left(e^{\gamma x}-1\right) \exp \left\{-\frac{\theta}{\gamma}\left(e^{\gamma x}-1\right)\right\} \theta}{\left[1-\exp \left\{-\frac{\theta}{\gamma}\left(e^{\gamma x}-1\right)\right\}\right] \gamma}, & J_{\theta b}=\frac{\partial^{2} \log }{\partial \theta \partial b}=-\frac{\left(e^{\gamma x}-1\right)}{\gamma}, \\
J_{\gamma a}=\frac{\partial^{2} \log }{\partial \gamma \partial a}=\frac{\theta \exp \left\{-\frac{\theta}{\gamma}\left(e^{\gamma x}-1\right)\right\}\left[e^{\gamma x}(x \gamma-1)+1\right]}{\gamma^{\wedge} 2\left[1-\exp \left\{-\frac{\theta}{\gamma}\left(e^{\gamma x}-1\right)\right\}\right]}, \quad J_{\gamma b}=\frac{\partial^{2} \log }{\partial \gamma \partial b}=-\frac{\left[e^{\gamma x}(x \gamma-1)+1\right] \theta}{\gamma^{2}}, \\
J_{\theta \theta}=\frac{\partial^{2} \log }{\partial \theta^{2}}=-\frac{1}{\theta^{2}}+\left(\frac{e^{\gamma x}-1}{\gamma}\right)^{2} \frac{(a-1) \exp \left\{-\frac{\theta}{\gamma}\left(e^{\gamma x}-1\right)\right\}}{\left[1-\exp \left\{-\frac{\theta}{\gamma}\left(e^{\gamma x}-1\right)\right\}\right]^{2},}
\end{array}
$$




$$
\begin{aligned}
& J_{\theta \gamma}=\frac{\partial^{2} \log }{\partial \theta \partial \gamma}=-\frac{e^{\gamma x}(x \gamma-1)+1}{\gamma^{2}}\left(\frac{(a-1) \exp \left\{-\frac{\theta}{\gamma}\left(e^{\gamma x}-1\right)\right\}}{\left[1-\exp \left\{-\frac{\theta}{\gamma}\left(e^{\gamma x}-1\right)\right\}\right]}-b\right), \\
& J_{\gamma \theta}=\frac{\partial^{2} \log }{\partial \theta^{2}}=\left\{b+\frac{(a-1) \exp \left\{-\frac{\theta}{\gamma}\left(e^{\gamma x}-1\right)\right\}}{\left[1-\exp \left\{-\frac{\theta}{\gamma}\left(e^{\gamma x}-1\right)\right\}\right]}+\frac{(a-1) \theta \exp \left\{-\frac{\theta}{\gamma}\left(e^{\gamma x}-1\right)\right\}}{\gamma\left[1-\exp \left\{-\frac{\theta}{\gamma}\left(e^{\gamma x}-1\right)\right\}\right]^{2}}\right\} \\
& \times\left[\frac{e^{\gamma x}(x \gamma-1)+1}{\gamma^{2}}\right], \\
& J_{\gamma \gamma}=\frac{\partial^{2} \log }{\partial \gamma^{2}}=\left\{\frac{x^{2} y^{2} e^{\gamma x}}{\gamma^{3}} \frac{(a-1) \theta \exp \left\{-\frac{\theta}{\gamma}\left(e^{\gamma x}-1\right)\right\}}{\gamma\left[1-\exp \left\{-\frac{\theta}{\gamma}\left(e^{\gamma x}-1\right)\right\}\right]^{2}}\right\}\left[\frac{(a-1) \theta \exp \left\{-\frac{\theta}{\gamma}\left(e^{\gamma x}-1\right)\right\}}{\left[1-\exp \left\{-\frac{\theta}{\gamma}\left(e^{\gamma x}-1\right)\right\}\right]}\right] \\
& +\left[\frac{e^{\gamma x}(x \gamma-1)+1}{\gamma^{2}}\right]\left\{\frac{(a-1) \theta^{2} \exp \left\{-\frac{\theta}{\gamma}\left(e^{\gamma x}-1\right)\right\}\left[e^{\gamma x}(x \gamma-1)+1\right]}{\gamma^{2}\left[1-\exp \left\{-\frac{\theta}{\gamma}\left(e^{\gamma x}-1\right)\right\}\right]^{2}}\right\} .
\end{aligned}
$$




\section{References}

[1] Cordeiro, G.M. and de Castro, M. A new family of generalized distributions. Journal of Statistical Computation and Simulation 81.7 (2011): 883-898.

[2] El-Gohary, A., Alshamrani, A. and Al-Otaibi,A.N. The generalized Gompertz distribution. Applied Mathematical Modelling 37.1 (2013): 13-24.

[3] Eugene, N., Lee, C. and Famoye, F. Beta-normal distribution and its applications. Communications in Statistics - Theory and Methods 31.4 (2002): 497-512.

[4] Gradshteyn, I. S. and Ryzhik, I. M. Tables of integrals, series, and products. New York: Academic Press (2000).

[5] Jones, M.C. Kumaraswamy's distribution: A beta-type distribution with some tractability advantages. Statistical Methodology 6.1 (2009): 70-81.

[6] Kenney, J.F. and Keeping, E.S. Mathematics of Statistics, part 1. Princeton, NJ: Van Nostrand (1962): 101-102.

[7] Kumaraswamy, P. A generalized probability density function for double-bounded random processes. Journal of Hydrology 46.1 (1980): 79-88.

[8] Kunimura, D. The Gompertz distribution-estimation of parameters. Actuarial Research Clearing House 2 (1998): 65-76.

[9] Marshall, A.W. and Olkin, I. Life distributions: Structure of nonparametric, semiparametric and parametric families. Springer (2007).

[10] Milgram, M. The generalized integro-exponential function. Mathematics of Computation 44.170 (1985): 443-458.

[11]Nadarajah, S., Cordeiro, G.M. and Ortega, E.M.M. General results for the Kumaraswamy-G distribution. Journal of Statistical Computation and Simulation 82.7 (2012): 951-979.

[12]Pollard, J.H. and Valkovics, E.J. The Gompertz distribution and its applications. Genus 48(3-4) (1992): 15-28.

[13]Rushton, J. P. and Templer, D.I. National differences in intelligence, crime, income, and skin color. Intelligence 37.4 (2009): 341-346.

[14] Shannon, C.E. A mathematical theory of communication. Bell System Technical Journal 27 (1948): 379-423. 
[15]Rényi, A. On measures of entropy and information. Proceedings of the Fourth Berkeley Symposium on Mathematics, Statistics and Probability (1961): 547-561.

[16]Willekens, F. Gompertz in context: The Gompertz and related distributions. Springer Netherlands (2002).

[17]Willemse, W. J. and Koppelaar, H. Knowledge elicitation of Gompertz'law of mortality. Scandinavian Actuarial Journal 2 (2000): 168-179.

Received March 15, 2013; accepted November 10, 2013.

\author{
Raquel C. da Silva. \\ Departamento de Estat'1stica, \\ Universidade Federal de Pernambuco, \\ 50740-540, Recife, PE,Brazil \\ e-mail:raquelmendiola@yahoo.com.br
}


